\title{
Outpatient versus inpatient follow-up for intrahepatic cholestasis of pregnancy
}

\author{
Ozgur Ozyuncu', Gokcen Orgul', Gonca Ozten'1, Murat Yurdakok², Mehmet Sinan Beksac \\ 'Department of Obstetrics and Gynecology, Division of Perinatology, Hacettepe University Faculty of Medicine, Ankara, Turkey \\ ${ }^{2}$ Department of Pediatrics, Division of Neonatology, Hacettepe University Faculty of Medicine, Ankara, Turkey
}

\begin{abstract}
Aim of the study: The optimum management method and the best time of delivery still remain unclear for intrahepatic cholestasis of pregnancy (ICP). We aimed to ascertain whether there is a benefit of close monitoring at hospital. Material and methods: We evaluated the maternal and neonatal records of ICPs over a recent five-year period. A total of 35 women and their 38 newborns were analyzed. The impact of hospitalization was evaluated in terms of delivery type, labor induction, preterm delivery, Apgar scores, and neonatal intensive care unit admission.

Results: The median maternal age was 30.7 years, and median gestation at diagnosis was 34 weeks. Alanine aminotransferase (ALT) and aspartate aminotransferase (AST) levels were normal in three patients; 32 (91.4\%) women had at least one increased hepatic enzyme level. The median AST level was elevated to $66 \mathrm{U} / \mathrm{l}$ (almost two-fold), and the median ALT level was $91 \mathrm{U} / \mathrm{l}$ (about three-fold). The median gestation time at delivery was 37 weeks. The preterm delivery rate was $45.7 \%$. The median birth weight was $3020 \mathrm{~g}$, and median Apgar scores were 9 and 10 at the $1^{\text {st }}$ and $5^{\text {th }}$ minutes, respectively. Neonatal intensive care unit admission occurred in 21 neonates (55.3\%). During pregnancy follow-up, 22 women (62.8\%) were hospitalized during their pregnancies. There was no statistically significant difference between groups in terms of obstetric and perinatal outcomes $(p>0.05)$. However, the hospitalized pregnant women were found to have higher levels of serum transaminases ( $p=0.15$ and $p=0.01$ for ALT and AST, respectively).
\end{abstract}

Conclusions: Hospitalization may be helpful in some ICP cases, especially when enzyme levels are elevated.

Key words: pregnancy, intrahepatic cholestasis, hospitalization.

\section{Address for correspondence}

Gokcen Orgul, Department of Obstetrics and Gynecology, Division of Perinatology, Hacettepe University, Faculty of Medicine, No. 81, 06100 Sihhiye, Ankara, Turkey, phone: +905556066254, e-mail: gokcenorgul@gmail.com

\section{Introduction}

Intrahepatic cholestasis of pregnancy (ICP) is an important liver disorder in pregnancy, and usually occurs during the second or third trimester $[1,2]$. Elevated serum total bile acid levels $(\geq 10 \mu \mathrm{mol} / \mathrm{l})$ and increased serum transaminases with pruritus are the typical characteristic findings of this pregnancy-specific disease $[1,2]$. The general incidence of ICP has been reported to be $0.1 \%$ to $2 \%$, and it is more frequent (up to 15\%) in some ethnic regions [3].

The underlying reasons for ICP have not been determined yet. However, environmental, immuno- logical, metabolic, and hormonal factors prompting inflammatory processes are thought to be the main etiological reasons behind the condition $[4,5]$. The relationship between adverse fetal outcome and serum bile acid levels has been investigated in previous studies $[1,6]$. Increased perinatal morbidity and mortality have been reported in pregnancies with gestational intrahepatic cholestasis $[1,7]$.

A multilevel classification system for ICP has been described in terms of serum bile acid levels, as follows: mild (10-40 $\mu \mathrm{mol} / \mathrm{l})$, moderate $(40-100 \mu \mathrm{mol} / \mathrm{l})$ or severe $(>100 \mu \mathrm{mol} / \mathrm{l})[1,8]$. Treatment is critical to reduce maternal symptoms, and ursodeoxycholic acid 
(UDCA) is the treatment of choice in routine clinical practice $[4,5,9]$. Obstetric complications such as preterm labor, meconium stained amniotic fluid, acute fetal distress, and/or stillbirth are all potential complications that are correlated with ICP $[8,10]$. The optimum time to deliver the baby is also not clear, even though the majority of relevant study authors suggest inducing labor between the $36^{\text {th }}$ and $37^{\text {th }}$ gestational week $[1,10,11]$.

This topic remains one of the most important dilemmas in perinatal medicine, and still needs to be verified objectively. In the present study, we discuss our management strategies for pregnancies seen together with ICP.

\section{Material and methods}

In this retrospective study, we evaluated the records of women diagnosed with ICP from January 2010 to December 2015. All data, including maternal and fetal variables, were obtained from the medical files of the patients and the computerized hospital system of Hacettepe University Hospitals. ICP diagnosis was carried out based on new onset pruritus with a total bile acid level $>10 \mu \mathrm{mol} / \mathrm{l}$ without any additional liver diseases. ICP patients with a serum bile acid level between 10 and $100 \mu \mathrm{mol} / \mathrm{l}$ were defined as the study group. We excluded patients who had an extremely high level of bile acid (> $100 \mu \mathrm{mol} / \mathrm{l})$, which was defined as severe ICP in previous studies $[1,8]$, because the gestational time for labor induction of these women is complicated, and there is no consensus about the optimum management.

Maternal follow-up was performed at our perinatology department after definitive diagnosis of ICP. UDCA was prescribed to all patients immediately until delivery. Patients were monitored by serial laboratory tests, especially alanine aminotransferase (ALT) and aspartate aminotransferase (AST) assessment, once a week. However, the screenings were more frequent, even daily or every second day, in cases of a worsening clinical condition or elevated ALT/AST levels. The highest liver function test results $(\geq 35 \mathrm{U} / \mathrm{l})$ were noted for each patient separately. Additionally, the measurements of serum hematocrit and hemoglobin levels (before and after birth), which is a standard procedure completed for every delivery in our hospital, were analyzed to understand the blood loss at birth.

Information about maternal age, obstetric history, previous ICP history, and comorbid diseases (if any) was retrieved from the maternal medical files. Patients were hospitalized for only a better and closer follow-up. Indications for the maternal hospitalization were extremely high ALT/AST levels, maternal anxiety, socioeconomic reasons and clinician awareness. We evaluated the maternal length of hospital stay during pregnancy and after delivery.

Fetal movement counting, ultrasonographic examination with Doppler, and a non-stress test were used for perinatal surveillance in ICP cases. The necessary data about delivery type (vaginal or cesarean section [CS]) and timing of delivery were retrieved. The medical records were also retrieved to evaluate CS indications, and only one reason was accepted for each individual. The gestational week at delivery was analyzed to understand the relation between preterm labor and ICP. Preterm birth was defined as labor occurring before the $37^{\text {th }}$ gestational week. Labor induction was considered in cases involving any clinical suspicion for increased maternal or fetal risk.

Perinatal outcome was the other concern of our study, and related data were retrieved from the medical files of the newborns. Birth weight, gender, Apgar score, neonatal intensive care unit admission (with reasons), and length of hospitalization were evaluated. The first assessment of the neonates was performed immediately in the delivery room by pediatricians. Further evaluation and interventions were carried out at our neonatology department.

The study was approved by Hacettepe University Non-Interventional Ethics Committee (GO 16/10022.03.2016) and conducted according to the Declaration of Helsinki. All statistical analyses were performed using SPSS software (IBM-SPSS, Inc., Chicago, IL, USA), version 23. The mean and standard deviation values along with the median, minimum, and maximum values were calculated, and the percentages were calculated where necessary. Normality of the data distribution was assessed with the Kolmogorov-Smirnov test. Parametric data were examined with independent two-sample or $t$ test, and non-parametric data were compared using the Mann-Whitney $U$ test. Percentages were compared with the chi-square test. A $p$ value $<0.05$ indicates statistically significant differences.

\section{Results}

A total of 35 women and their 38 newborns were included in this study. Table 1 shows the main demographic and characteristic findings of the study group. The median maternal age was 30.7 years (range, $21-49$ years), and the median gestation at diagnosis was 34 weeks (range, 27-40 weeks) in patients with ICP. There were 32 singleton pregnancies with three sets of twin pregnancies. In these multiple pregnancies, two out of three were in vitro fertilization pregnancies. Of the 35 women, 13 were 
nulliparous, while the remaining 22 were multiparous. Two patients had a history of ICP in their previous pregnancies. Relevant comorbid diseases, found in 10 patients (28.6\%), were as follows: gestational diabetes mellitus in 4, Hashimoto's thyroiditis in 3, familial hypercholesterolemia in 1, familial Mediterranean fever (FMF) in 1, and Crohn's disease in one.

There was only one case without UDCA treatment due to the late onset of symptoms, and delivery was planned at 40 weeks and one day after diagnosis. ALT and AST levels were normal in three patients; however, 32 (91.4\%) out of 35 women had at least one increased hepatic enzyme level. Median AST level was elevated to $66 \mathrm{U} / 1$ (almost two-fold), and median ALT level was $91 \mathrm{U} / 1$ (about three-fold) among the cases.

During pregnancy follow-up, 22 women (62.8\%) were hospitalized during their pregnancies (Table 2). The average length of stay was $5.5 \pm 6.7$ days among all patients. The longest stay was 25 days, during which the patient was hospitalized due to transportation difficulty, without any medical indication. Following delivery, the mothers were discharged on postpartum day 3 ( $2.5 \pm 0.9$ days). Neither maternal morbidity nor mortality occurred during pregnancy or the postpartum period. The impact of hospitalization was also evaluated in terms of delivery type, labor induction, preterm delivery, Apgar scores, and neonatal intensive care unit admission, but there was no significant difference between the groups. However, the hospitalized pregnant women were found to have higher levels of serum transaminases ( $p=0.15$ and $p=0.01$ for ALT and AST, respectively).

The median gestation time at delivery was 37 weeks (range, 32 4/7-40 1/7 weeks). Preterm delivery was detected in 16 pregnancies (four induced; oligohydramnios in 2, intrauterine growth retardation (IUGR) in 1 and preterm premature membrane rupture in 1 and 12 spontaneous), and the preterm delivery rate was found to be $45.7 \%$. Spontaneous preterm delivery occurred in four pregnancies, and mean gestational age was 35 weeks and 4 days in these cases.

In total, there were 23 women (65.7\%) in whom delivery was induced. Eleven of them delivered after the $37^{\text {th }}$ gestational week, while pregnancy was induced in 12 women before the $37^{\text {th }}$ gestational week. Overall, 36 weeks was found to be the median gestational time for these 23 pregnancies.

Four (11.4\%) vaginal deliveries and 31 (88.6\%) cesarean deliveries were recorded from the 35 pregnant women. CS indications were fetal distress in 11 (fetal heart rate abnormalities detected by electronic fetal monitoring in 8, abnormal Doppler ultrasonography findings in 2 IUGR fetuses and umbilical cord prolapse
Table 1. Maternal and fetal variables

\begin{tabular}{lcccc}
\hline & Mean (SD) & Median & Min & Max \\
\hline Maternal $(\boldsymbol{n}=35)$ & & & & \\
\hline Age & $30.7(6.4)$ & 30 & 21 & 49 \\
\hline Gravida & $2.1(1.1)$ & 2 & 1 & 5 \\
\hline Parity & $0.6(0.7)$ & 1 & 0 & 2 \\
\hline Abortion & $0.4(0.8)$ & 0 & 0 & 3 \\
\hline Living child & $0.5(0.7)$ & 0 & 0 & 2 \\
\hline Highest AST (U/I) & $102.8(99.7)$ & 66 & 7 & 467 \\
\hline Highest ALT (U/I) & $140.3(118.5)$ & 91 & 16 & 472 \\
\hline Hospitalization (day) & $5.5(6.7)$ & 3 & 0 & 25 \\
\hline Fetal $(\boldsymbol{n}=38)$ & & & & \\
\hline Birth weight (g) & $2977.4(550.6)$ & 3020 & 1460 & 4080 \\
\hline Apgar 1 & $8.8(0.7)$ & 9 & 6 & 10 \\
\hline Apgar 5 & $9.8(0.6)$ & 10 & 8 & 10 \\
\hline Hospitalization (day) & $3.4(4.5)$ & 3 & 0 & 18 \\
\hline
\end{tabular}

Table 2. Comparison of patients in terms of their hospitalization situation

\begin{tabular}{|c|c|c|c|}
\hline & $\begin{array}{l}\text { Not hospitalized } \\
\quad(n=13)\end{array}$ & $\begin{array}{l}\text { Hospitalized } \\
(n=22)\end{array}$ & $P$ value \\
\hline Highest ALT (mean) (U/I) & 80.2 & 175.8 & $0.015^{*}$ \\
\hline Highest AST (mean) (U/I) & 48 & 135.1 & $0.01 *$ \\
\hline $\begin{array}{l}\text { Number of labor } \\
\text { inductions }\end{array}$ & 6 & 17 & 0.065 \\
\hline $\begin{array}{l}\text { Number of preterm } \\
\text { deliveries }\end{array}$ & 6 & 10 & 0.599 \\
\hline $\begin{array}{l}\text { Vaginal delivery/ } \\
\text { cesarean section }\end{array}$ & $\begin{array}{c}2 \\
11\end{array}$ & $\begin{array}{c}2 \\
20\end{array}$ & 0.643 \\
\hline $\begin{array}{l}\text { Number of neonatal care } \\
\text { unit admissions }\end{array}$ & 5 & 14 & 0.113 \\
\hline Gestational day at birth & 261 & 259 & 0.291 \\
\hline Birth weight (g) & 3120 & 2917 & 0.356 \\
\hline Apgar $1^{\text {st }}$ minute & 8.5 & 8.9 & 0.389 \\
\hline Apgar $5^{\text {th }}$ minute & 9.6 & 9.8 & 0.489 \\
\hline
\end{tabular}

*statistically significant

in 1), breech presentation in two, previous CS history in 9, failure to progress during labor in six, and twin pregnancies in three, out of the total 31 . Blood loss was calculated by means of decreases in hemoglobin and hematocrit levels from pregnancy to after delivery. Decreases in hemoglobin and hematocrit levels were $1.3 \mathrm{~g} / \mathrm{dl}$ and $5 \%$ after delivery, respectively. There were only three cases of postpartum bleeding (around $1000 \mathrm{ml}$ ) (8.5\%); however, erythrocyte transfusion was not required in any of them. 
There were 25 male and 13 female infants out of the 35 pregnancies (counting each set of twins as one pregnancy) diagnosed with ICP. We did not observe any perinatal mortality, meconium stained amniotic fluid, or congenital malformation among the neonates. The median birth weight was $3020 \mathrm{~g}$ (range, 1460-4080 g), and median Apgar scores were 9 and 10 at the $1^{\text {st }}$ min and $5^{\text {th }}$ min after birth, respectively. There were only three (7.9\%) newborns with an Apgar score $\leq 7$ at $1 \mathrm{~min}$; however, no newborn was recorded to have an Apgar score $\leq 7$ at $5 \mathrm{~min}$.

Neonatal intensive care unit admission occurred in 21 neonates (55.3\%). The mean length of hospital stay was 6 days (2-18 days) for these 21 hospitalized babies. Indications were prematurity in eight, respiratory system problems in five, jaundice in two, and a requirement of follow-up in the remaining six babies.

\section{Discussion}

The genetic etiology of ICP has been investigated in previous studies. There is no genetic locus directly related to ICP; however, some mutations (multidrug resistance 3-, adenosine triphosphate binding cassette) have been reported to be associated with the disease $[10,12]$.

It has been reported that inflammation is responsible for hepatocyte injury, altered apoptosis, biliary transport system dysfunction, and increased bile acid toxicity $[4,5,13]$. Inflammation seems to be a risk factor for etiological pathology in the occurrence of ICP. Similarly, inflammation-related hepatic cell degradation products are probably responsible for the injury of cellular components of the intervillous space, which is very important in maternal-fetal interaction. These biological events may be the reason for obstetrical complications such as prematurity, preeclampsia, and chronic impaired fetal perfusion.

Women with ICP carry a high risk for preeclampsia and gestational diabetes mellitus (GDM) in their pregnancies $[7,14,15]$. We observed that 4 out of 35 (11.4\%) pregnancies were complicated with GDM. On the other hand, there was no preeclampsia in our small study group. It has also been reported that future onset of chronic inflammatory diseases (psoriasis, Crohn's disease) and hepatic cancer risk are increased in women with an ICP history [2]. In our series, there were five cases $(14.2 \%)$ with coexisting autoimmune diseases, Hashimoto thyroiditis in three, Crohn's disease in one, and FMF in one.

Women with ICP are also at a higher risk of cardiovascular disease morbidity [2]. There was one case of comorbid familial hypercholesterolemia in our study group. It is well known that women with familial hypercholesterolemia also have a greater risk of cardiovascular disease development [16]. We can thus speculate that cardiac events may be related to impaired cholesterol mechanisms in ICP, which is responsible for endothelial injury and atherosclerosis. Blood lipid panel and cholesterol testing may be offered to highrisk women with ICP at the postpartum period, when pregnancy specific hormones lose their effect on plasma lipid profiles.

The management of pregnancies with ICP is difficult, and there is no accepted consensus about the optimum time to deliver the baby. Obstetricians remain largely confused between the need to avoid iatrogenic prematurity and mitigating the risk of intrauterine death by waiting for fetal maturity. There are also other adverse perinatal outcomes related to ICP, which include preterm delivery, meconium-stained amniotic fluid, and respiratory distress syndrome $[1,7,8]$. In this study, labor induction was performed in 23 women (65.7\%). There were no fetal/neonatal deaths, meconium stained amniotic fluid, or congenital malformation. We have shown that a successful pregnancy outcome is possible with high rates of labor induction. However, it is impossible to say whether an expectant management strategy would be harmful for all fetuses if we had waited for the spontaneous onset of labor.

Mode of delivery is also another issue for these high-risk pregnancies. Elective CS is not a routine procedure, and vaginal delivery must be the first option [17]. In this study, we had a significantly higher rate of CS than typical, mostly performed for obstetrical indications. Additionally, maternal request for CS and anxiety are more common among women who are informed about potential complications. There were no cases of CS-related morbidity in our patients, and blood loss was within the normal range. It has been reported that postpartum hemorrhage risk is not increased among women with ICP [18], and our findings support this information.

Studies are generally focused on the prediction of rational management in order to prevent perinatal morbidity and mortality. Thus, the results of the different studies carried out by studying the time of disease onset, maternal serum transaminases levels and biophysical profile scores are confusing. The best approach in routine practice seems to be serum bile acid level measurements (cut-off levels are debatable) [1]. In this study, we excluded patients with extremely high bile acid levels, and our successful pregnancy outcomes are probably due to this approach. Hence, it can be concluded that active management is necessary to have a better clinical outcome. 
Prematurity and respiratory distress are the main problems for newborns $[1,11]$. In our study, these two complications were detected in 13 of 38 (34.2\%) cases; however, resuscitation requirement was not observed among our cases after birth, and Apgar scores were satisfactory for all babies. In conclusion, pediatricians should be well informed to take precautions and prevent possible morbidity.

In conclusion, increased perinatal morbidity and mortality are the main concerns of many physicians. Intensive care is necessary to prevent preterm deliveries and maternal complaints. Hospitalization may be helpful in some cases, especially when the enzyme levels are elevated. However, the benefit of close monitoring at the hospital is not clear, and each patient should be evaluated separately.

\section{Disclosure}

The authors report no conflict of interest.

\section{References}

1. Brouwers L, Koster MP, Page-Christiaens GC, et al. Intrahepatic cholestasis of pregnancy: maternal and fetal outcomes associated with elevated bile acid levels. Am J Obstet Gynecol 2015; 212: 100. e1-7.

2. Wikström Shemer EA, Stephansson O, Thuresson M, et al. Intrahepatic cholestasis of pregnancy and cancer, immune-mediated and cardiovascular diseases: A population-based cohort study. J Hepatol 2015; 63: 456-461.

3. Williamson C, Geenes V. Intrahepatic cholestasis of pregnancy. Obstet Gynecol 2014; 124: 120-133.

4. Biberoglu E, Kirbas A, Daglar K, et al. Role of inflammation in intrahepatic cholestasis of pregnancy. J Obstet Gynaecol Res 2016; 42: 252-257.

5. Webb GJ, Elsharkawy AM, Hirschfield GM. Editorial: the etiology of intrahepatic cholestasis of pregnancy: towards solving a monkey puzzle. Am J Gastroenterol 2014; 109: 85-88.

6. Geenes V, Chappell LC, Seed PT, et al. Association of severe intrahepatic cholestasis of pregnancy with adverse pregnancy outcomes: A prospective population-based case-control study. Hepatology 2014; 59: 1482-1491.

7. Wikström Shemer E, Marschall H, Ludvigsson J, Stephansson O. Intrahepatic cholestasis of pregnancy and associated adverse pregnancy and fetal outcomes: a 12-year population-based cohort study. BJOG 2013; 120: 717-723.

8. Kawakita T, Parikh LI, Ramsey PS, et al. Predictors of adverse neonatal outcomes in intrahepatic cholestasis of pregnancy. Am J Obstet Gynecol 2015; 213: 570.e1-8.

9. Abu-Hayyeh S, Papacleovoulou G, Lövgren-Sandblom A, et al. Intrahepatic cholestasis of pregnancy levels of sulfated progesterone metabolites inhibit farnesoid $\mathrm{X}$ receptor resulting in a cholestatic phenotype. Hepatology 2013; 57: 716-726.

10. Ozkan S, Ceylan Y, Ozkan OV, Yildirim S. Review of a challenging clinical issue: Intrahepatic cholestasis of pregnancy. World J Gastroenterol 2015; 21: 7134-7141.

11. Puljic A, Kim E, Page J, et al. The risk of infant and fetal death by each additional week of expectant management in intrahepatic cholestasis of pregnancy by gestational age. Am J Obstet Gynecol 2015; 212: 667.e1-5.

12. Mella MT, Kohari K, Jones R, et al. Mitochondrial gene expression profiles are associated with intrahepatic cholestasis of pregnancy. Placenta 2016; 45: 16-23.

13. Ersoy AO, Kirbas A, Ozler S, et al. Maternal and fetal serum levels of caspase-cleaved fragments of cytokeratin-18 in intrahepatic cholestasis of pregnancy. J Matern Fetal Neonatal Med 2016; 29: 562-566.

14. Martineau M, Raker C, Powrie R, et al. Intrahepatic cholestasis of pregnancy is associated with an increased risk of gestational diabetes. Eur J Obstet Gynecol Reprod Biol 2014; 176: 80-85.

15. Raz Y, Lavie A, Vered Y, et al. Severe intrahepatic cholestasis of pregnancy is a risk factor for preeclampsia in singleton and twin pregnancies. Am J Obstet Gynecol 2015; 213: 395.e1-8.

16. Wong B, Kruse G, Kutikova L, et al. Cardiovascular disease risk associated with familial hypercholesterolemia: a systematic review of the literature. Clin Ther 2016; 38: 1696-1709.

17. Lo JO, Shaffer BL, Allen AJ, et al. Intrahepatic cholestasis of pregnancy and timing of delivery. J Matern Fetal Neonatal Med 2015; 28: 2254-2258

18. Furrer R, Winter K, Schäffer L, et al. Postpartum blood loss in women treated for intrahepatic cholestasis of pregnancy. Obstet Gynecol 2016; 128: 1048-1052. 